with a lightning flash. Eckersley furthermore describes trains of whistlers having a time separation of about 3.8 sec. between each succeeding whistler. It seems probable, therefore, that the phenomena of multiple signal bursts and whistlers are closely associated.

A more detailed account of these observations will be published later.

Research Division,

G. A. Isted

Marconi's Wireless Telegraph Co., Ltd. Jan. 21.

${ }^{1}$ Nature, 122, 768 (1928).

\section{A Theoretical Model for Arches in the Solar Corona}

IN recent years, the belief has grown among solar physicists that magnetic fields play an essential part in the phenomena of solar prominences. The study of the static equilibrium of an ionized atmosphere in a gravitational field with some magnetic field also present is expected to shed light on the more difficult dynamical problems, but may also provide models for quiescent prominences, which appear to be almost in static equilibrium. The condition for static equilibrium is that the resultant of the pressure gradient and the gravitational and electromagnetic force densities should vanish.

The simple model has been studied in which (i) the temperature and composition of the atmosphere are uniform; (ii) the gravitational field is uniform, the $z$-axis pointing upwards ; and (iii) the magnetic field is two-dimensional, that is, $H_{y}=0 ; \partial H_{x} / \partial y=$ $\partial H_{z} / \partial y=0$; the current density $\mathbf{j}$ then has only a $y$-component.

The condition for static equilibrium can only be satisfied by magnetic fields for which $\mathbf{j} \exp z / h$ is constant on a line of force, where $h$ is the scale height. Civen such a field, the pressure is determined apart from an arbitrary constant. A particularly useful family of solutions is provided by a set of lines of force, such that any magnetic field having these lines of force satisfies the above condition.

These lines of force are the lines of constant $F$, where

$$
F=\exp (-z / h)-2 \exp (-z / 2 h) \cos (x / 2 h)+1
$$

and are shown in the diagram. For $F<1$, they are closed curves, and the advantage of this family of solutions is that the magnetic field can be taken to vanish outside one of these closed lines of force, so avoiding any difficulty with boundary conditions. The horizontal width of the closed curves does not exceed $2 \pi h$.

The effect of the magnetic field on the gas pressure $p$ can be expressed in terms of the magnetic pressure $p_{H}$ given by:

$$
p_{H}=H^{2} / 8 \pi \text {. }
$$

Both $p \exp z / h$ and $p_{H} \exp z / h$ are constant on a line of force for this family of solutions, and the variation of pressure is given by :

$$
\frac{\mathrm{d}}{\mathrm{d} F}\left[\{\exp (z / h)\}\left(p+p_{H}\right)\right]=(-\exp z / h) p_{H} / F,
$$

which shows that $(\exp z / h)\left(p+p_{H}\right)$ increases towards the inside of the closed curves.

The two-dimensional form of these solutions makes them appropriate for those prominences which

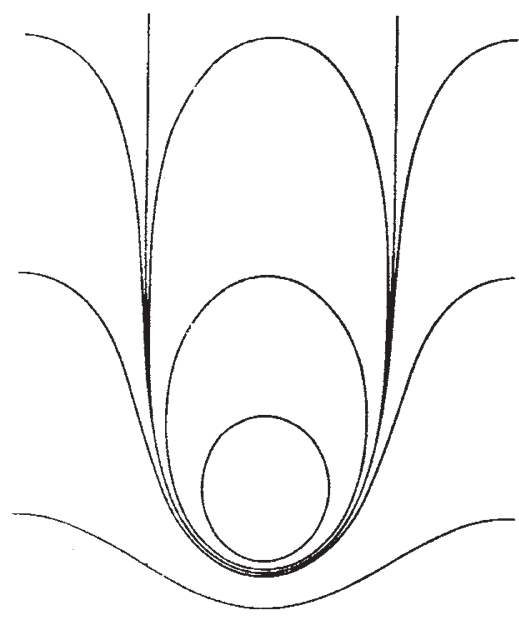

appear as filaments on the disk of the sun, and the effect expressed by (3) may explain the high pressure of prominences relative to the surrounding gas; but, because prominences are much cooler than the corona, the isothermal model must be seriously wrong in some respects. There seems to be much better justification for applying this model to the arches seen in the corona above prominences, as these may well be nearly isothermal. They are seen in white light at eclipses and are difficult to photograph; but a drawing reproduced in the "Handbuch der Astrophysik", 4, 200 (1929), shows arches which resemble the theoretical curves. The outer arches appear to be near the limiting width, $2 \pi h$; the width of the outer arch is about a quarter of the solar radius, and the temperature required to make these fit is about $10^{6 \circ} \mathrm{K}$., which is the accepted value for the temperature in the corona.

School of Physics,

J. W. Dungey

University of Sydney.

Oct. 2.

\section{The Lorentz Transformation}

$\mathrm{O}_{N}$ the basis of rather less restrictive postulates concerning the behaviour of light than are customary in special relativity theory, Capildeo has recently ${ }^{1}$ given the transformation equations

$$
\alpha x^{\prime}=x-V t, \quad y^{\prime}=y, \quad \begin{aligned}
z^{\prime} & =z, \\
\alpha t^{\prime} & =t-V x / c^{2},
\end{aligned}
$$

connecting two frames of reference $S$ and $S^{\prime}$ (with suitably oriented axes) in uniform relative motion, where, it is suggested, the value of $\alpha$ cannot be uniquely determined without additional evidence. If, however, we consider three frames of reference $S, S_{1}, S_{2}$, where the velocities of $S_{1}, S_{2}$ relative to $S$ are $V_{1}, V_{2}$ along $O x, O y$, respectively, then, with the obvious notation,

$$
\alpha_{1} x_{1}=x-V_{1} t, \quad y_{1}=y, \begin{gathered}
z_{1}=z, \\
\alpha_{1} t_{1}=t-V_{1} x / c^{2},
\end{gathered}
$$

and

$x_{2}=x, \quad \alpha_{2} y_{2}=y-V_{2} t, \quad z_{2}=z, \quad \alpha_{2} t_{2}=t-V_{2} y / c^{2}$, and it will be found after rotating the $x$-axes of $S_{1}$ and $S_{2}$ into the direction of their mutual relative 